\title{
Time Series Prediction Using Fuzzy Cerebellar Model Articulation Controller with Symbiotic Particle Swarm Optimization
}

\author{
Chin-Ling Lee and Cheng-Jian Lin
}

\begin{abstract}
In this paper, a fuzzy cerebellar model articulation controller (FCMAC) model with learning ability is proposed for solving the time series prediction problem. An efficient learning algorithm, called symbiotic particle swarm optimization (SPSO), combined symbiotic evolution and modified particle swarm optimization for tuning parameters of the FCMAC. Simulation results show that the converging speed and root mean square error (RMS) of the proposed method has a better performance than those of other methods.
\end{abstract}

Index Terms-Cerebellar model articulation controller, fuzzy set, particle swarm optimization, symbiotic evolution, time series, prediction.

\section{INTRODUCTION}

The cerebellar model articulation controller (CMAC) [1], developed by Albus, is a simple network architecture which provides the advantages of fast learning and a high convergence rate. The CMAC model has been successfully applied to various fields [2], [3].

Training of the parameters is the main problem in designing a CMAC model. Genetic algorithms (GAs), powerful tools based on biological mechanisms and natural selection theory [4], have received considerable attention regarding its potential as an optimization technique for complex problems and have been successfully applied in various areas [5]. A genetic algorithm (GA) is a parallel, global search technique that emulates operators. Because it simultaneously evaluates many points in the search space, it is more likely to converge toward the global solution. But the genetic algorithm has two main drawbacks. One is lack of the local search ability and the other is the premature convergence [6]. Therefore, in recent years, some researchers [6], [7] have proposed various improved-GAs to solve global optimization problems. In 1995, a new optimization algorithm, called particle swarm optimization (PSO) was developed by Kennedy and Eberhart [8]. The underlying motivation for the development of PSO algorithm is the social behavior of animals, such as bird flocking, fish

Manuscript received October 13, 2012; revised January 13, 2013. This work was supported in part by the National Science Council of the Republic of China, Taiwan for financially supporting this research under Contract No. NSC 101-2221-E-167-037 and No. 101-2622-E-167-005-CC3.

C. L. Lee is with the International Trade Department, National Taichung University of Science and Technology, Taichung City, Taiwan 404, ROC. (e-mail: merrylee@nutc.edu.tw).

C. J. Lin is with the Computer Science and Information Engineering Department, National Chin-Yi University of Technology, Taichung City, Taiwan 411, ROC. (e-mail:cjlin@ncut.edu.tw). schooling and swarm theory. The PSO has come to be widely used as a problem solving method in engineering and computer science. It is not only a recently invented high-performance optimizer that is very easy to understand and implement, but also requires less computational bookkeeping. But the PSO have one main drawback that convergence speed is too slow. Therefore, in this study, we proposed a symbiotic particle swarm optimization algorithm (SPSO) for solving the time series problems.

\section{The Structure of FuZZy Cerebellar Model ARTICULATION CONTROLLER}

In this paper, we propose a fuzzy CMAC (FCMAC) model. The FCMAC model [9], illustrated in Fig. 1, consists of the input space partition, association memory selection, and defuzzification. The FCMAC model is like the traditional CMAC model that approximates a nonlinear function $\mathrm{y}=\mathrm{f}(\mathrm{x})$ by using two primary mappings:

$$
\begin{aligned}
& S: X \Rightarrow A \\
& P: A \Rightarrow D
\end{aligned}
$$

where $X$ is a s-dimensional input space, $A$ is a $N_{A}$-dimensional association space, and $D$ is a one-dimensional output space. These two mappings are realized by using fuzzy operations. The function $S(x)$ maps each point $\mathrm{x}$ in the input space onto an association vector $\alpha=S(x)$ A that has NL nonzero elements $\left(N_{L}<N_{A}\right)$. Here, $\alpha=\left(\alpha_{1}, \alpha_{2}, \cdots, \alpha_{N_{A}}\right)$, where $0 \leq \alpha \leq 1$ for all components in is derived from the composition of the receptive field functions and sensory inputs. Different from the traditional CMAC model, several hypercubes are addressed by the input state $x$. The hypercube values are calculated by product operation through the strength of the receptive field functions for each input state.

In the FCMAC model, we use the Gaussian basis function as the receptive field function and the fuzzy weight function for learning. Some learned information is stored in the fuzzy weight vector. The one-dimension Gaussian basis function can be given as follows:

$$
\mu(x)=e^{-((x-m) / \sigma)^{2}}
$$

where $x$ represents the specific input state, $m$ represents the corresponding center, and $\sigma$ represents the corresponding variance. 
Let us consider a $N_{D}$-dimensional problem. A Gaussian basis function with $N_{D}$ dimensions is given as follows:

$$
\alpha_{j}=\prod_{i=1}^{N_{D}} e^{-\left(\left(x_{i}-m_{i j}\right) / \sigma_{i j}\right)^{2}}
$$

where $\Pi$ represents the product operation, $\alpha_{j}$ represents the $j$ th element of the association memory selection vector, $x_{i}$ represents the input value of the $i$ th dimension for a specific input state $x, m_{i j}$ represents the center of the receptive field functions, $\sigma_{i j}$ represents the variance of the receptive field functions, and $N_{D}$ represents the number of the receptive field functions for each input state. The function $P(\alpha)$ computes a scalar output $y$ by projecting the association memory selection vector onto a vector of adjustable fuzzy weights. Each fuzzy weight is inferred to produce a partial fuzzy output using the value of its corresponding association memory selection vector as the input matching degree. The fuzzy weight is considered here so that the partial fuzzy output is defuzzified into a scalar output using standard volume-based centroid defuzzification [7], [9]. The term volume is used in a general sense to include multi-dimensional functions. For two-dimensional functions, the volume reduces to the area. If $v_{j}$ is the volume of the consequent set and $\xi_{j}$ is the weight of the scale $\alpha_{j}$, then the general expression for defuzzification is

$$
y=\frac{\sum_{j=1}^{N_{L}} \alpha_{j} w_{j}^{m} v_{j} \xi_{j}}{\sum_{j=1}^{N_{L}} \alpha_{j} v_{j} \xi_{j}}
$$

where $w_{j}^{m}$ is the mean value of the fuzzy weights and $N_{L}$ is the number of hypercube cells. The volume $v_{j}$ in this case is simply the area of the consequent weights, which are represented by Gaussian fuzzy sets. Therefore, $v_{j}=w_{j}^{\sigma} \sqrt{\pi}$, where $w_{j}^{\sigma}$ represents the variance of the fuzzy weights. If the weight $\xi_{j}$ is considered to be one, as in this work, then the actual output $y$ is derived as follows:

$$
y=\frac{\sum_{j=1}^{N_{L}} \alpha_{j} w_{j}^{m} w_{j}^{\sigma}}{\sum_{j=1}^{N_{L}} \alpha_{j} w_{j}^{\sigma}}
$$

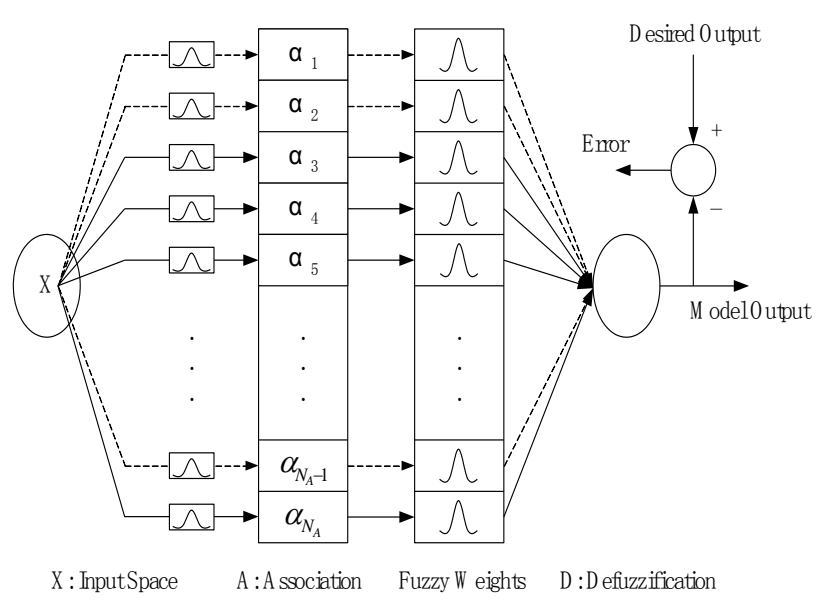

Fig. 1. The structure of the FCMAC model.

\section{LeARNING ALgORItHMS FOR THE FCMAC MOdEL}

This section describes the proposed evolutionary learning algorithm which is combine the symbiotic evolution and modified particle swarm optimization, called symbiotic particle swarm optimization (SPSO) algorithm. The notion of symbiotic evolution [10], [11] is similar to the implicit fitness sharing used in an immune system model. The authors evolve artificial antibodies to match or detect artificial antigens. Each antibody can only match a single antigen, and different antibodies are needed to protect effectively against various antigens. These antibodies consist in separating the population into subpopulations and changing the way fitness values are assigned by fitness sharing. An antibody is selected for replacement by randomly choosing a subset of the population and then selecting the member of that subset that is most similar to the new antibody. Therefore, summing the fitness values of all possible combinations of that antibody with other current antibodies and dividing the sum by the total number of combinations yields the fitness of an antibody.

The basic idea of symbiotic evolution is that an individual is used to represent a single fuzzy rule. A FCMAC model is formed when several individuals, which are randomly selected from a population, are combined. With the fitness assignment performed by symbiotic evolution, and with the local property of a fuzzy rule, symbiotic evolution and the FCMAC model design can complement each other. The structure of the proposed particles in the symbiotic evolution is shown in Fig. 2.

The learning process of the proposed SPSO includes the coding, initialization, fitness assignment and sub-particles update. The coding step is concerned with the membership functions and weights of a FCMAC model that represent particle for SPSO. The initialization step assigns the sub-particles values before the learning process begins. Then, the fitness value assignment gives a suitable value to each fuzzy rule during the learning process, the value higher the particles survive higher. Repeat until a given termination condition is met. The flowchart for parameter learning is shown in Fig. 3. The whole learning process is described step by step below.

\section{A. Production Initial Swarm}

The coding step is related with the membership functions and fuzzy rules of a FCMAC model that represent sub-particles suitable for symbiotic evolution. The initialization step must assign value before the evolution process begins. The initial value assigns by the randomly in the range $[-1,1]$ in each subgroup.

\section{B. Fitness Value Assign Step}

The fitness value of a rule (a sub-particle) is computed by summing up the fitness values of all the feasible combinations of that rule with all other randomly selected rules and then dividing the sum by the total number of combinations. The details for assigning the fitness value are described step by step as follows.

Step 1: Randomly select R fuzzy rules (sub-particle) from each subgroup for composing FCMAC model.

Step 2: Evaluate every FCMAC model that is generated from 
step 1 to obtain fitness value. The fitness value is designed according the follow formulation:

$$
F=\frac{1}{1+\sqrt{\frac{1}{D} \sum_{d=1}^{D}\left(y_{d}-\bar{y}_{d}\right)^{2}}}
$$

where $y_{d}$ represents the $d$ th model output; $\bar{y}_{d}$ represents the $d$ th desired output, and $D$ represents the number of input data. Step 3: Divide the fitness value by $R$ and accumulate the divided fitness value to the selected $R$ rules with their fitness value records that were set to zero initially.

Step 4: Repeat the above steps until each rule (sub-particle) in each subgroup has been selected a sufficient number of times, and record the number of FCMAC models in which each sub-particle has participated.

Step 5: Divide the accumulated fitness value of each sub-particle by the number of times it has been selected.

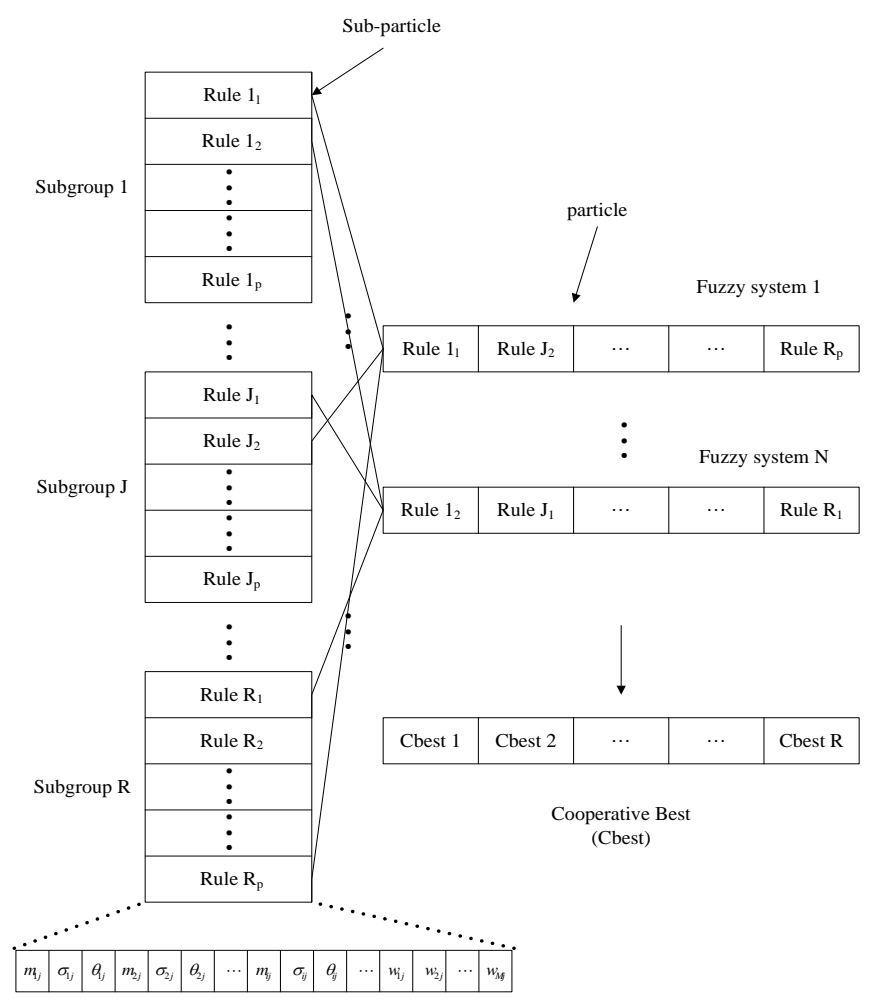

Fig. 2. Structure of the particles in the symbiotic evolution.

C. The Modified Particle Swarm Optimization (MPSO)

In this paper, we proposed the new velocity update function to move up the performance, the velocity of the sub-antibody is redefined as the following equation:

$$
\begin{aligned}
\vec{v}_{i}(k+1)= & \omega * \vec{v}_{i}(k)+\phi_{1} * \operatorname{rand}() *\left(\text { Lbest }-\vec{x}_{i}(k)\right) \\
& +\phi_{2} * \operatorname{rand}() *\left(\text { Gbest }-\overrightarrow{x_{i}}(k)\right)+\phi_{3} * \operatorname{rand}() *\left(\text { Cbest }-\vec{x}_{i}(k)\right)
\end{aligned}
$$

where $\omega, \phi_{1}, \phi_{2}$ and $\phi_{3}$ are called the coefficient of inertia, cognitive, group and society study respectively. The Cbest is changed according to rule. We hope that accelerate every sub-particle in a direction between the best self, the best of partial solution and the best of full solution so far.

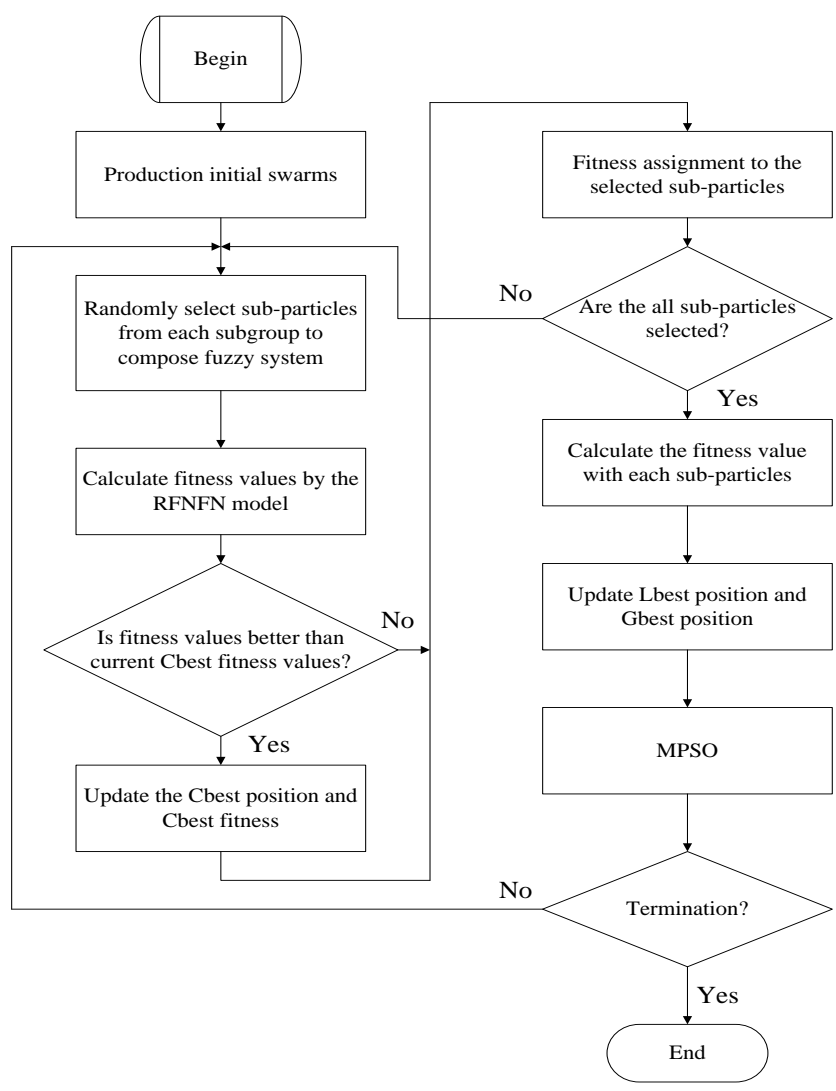

Fig. 3. Flowchart of the proposed SPSO designs method.

\section{Prediction of ChaOtic Time Series}

The Mackey-Glass chaotic time series $x(t)$ was generated using the following delay differential equation;

$$
\frac{d x(t)}{d t}=\frac{0.2 x(t-\tau)}{1+x^{10}(t-\tau)}-0.1 x(t)
$$

Crowder [12] extracted 1000 input-output data pairs $\left\{x, y^{d}\right\}$ using four past values of $x(t)$ :

$$
[x(t-18), x(t-12), x(t-6), x(t) ; x(t+6)]
$$

where $\tau=17$ and $x(0)=1.2$. Four inputs to the FCMAC model, corresponded to these values of $x(t)$, and one output was $x(t+\Delta t)$, where $\Delta t$ is a time interval into the future. The first 500 pairs (from $x(1)$ to $x(500)$ ) were the training data set, while the remaining 500 pairs (from $x(501)$ to $x(1000)$ ) were the testing data used to validate the proposed method. The parameter learning through SPSO method of the coefficient $w$ was set to 0.3 , the cognitive coefficient $\phi_{1}$ and the group coefficient $\phi_{2}$ were set to 1 , and the society coefficient $\phi_{3}$ was set to 1.5 . The swarm sizes were also set to 50 . The above-mentioned parameters $w 、 \phi_{1} 、 \phi_{2}$ and $\phi_{3}$, we are using the try-and-error methods to determine these parameter values.

In this example, we compared the performance of the 
SPSO learning algorithm with other methods. Fig. 4 shows the learning curves of the three methods. Figures 5(a)-(c) illustrate the prediction outputs of the various models. The errors between the desired output and model output are shown in Fig. 6 (a)-(c). As shown in Fig. 5 and 6, the prediction abilities of the proposed SPSO method were better than those of the PSO and GA methods.

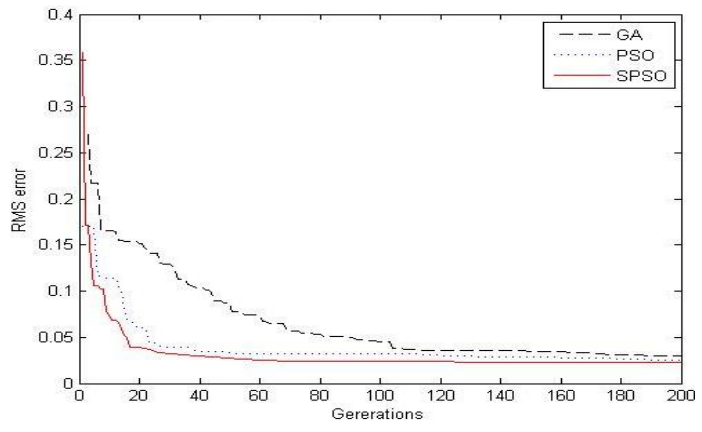

Fig. 4. Learning curves the proposed SPSO, the PSO and the GA methods.

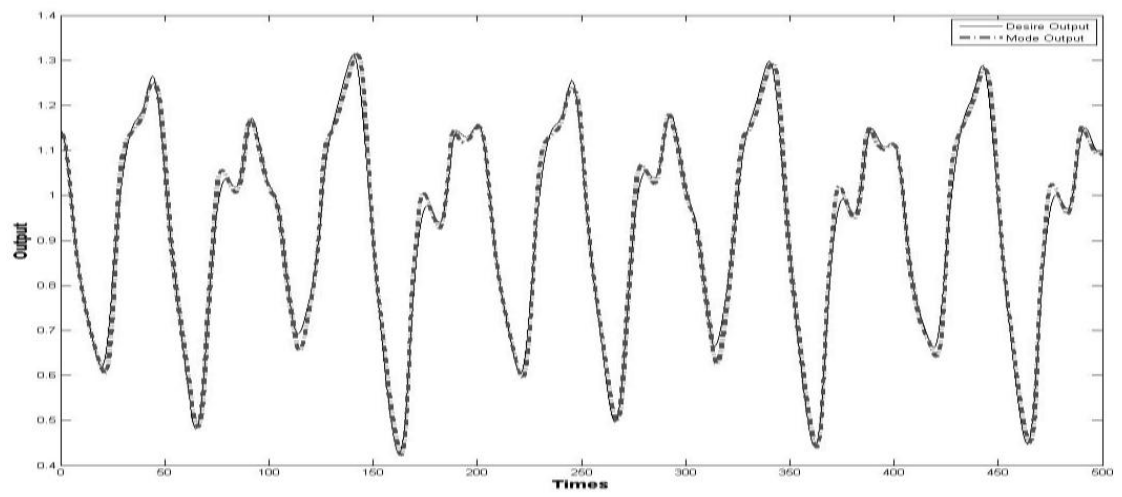

(a)

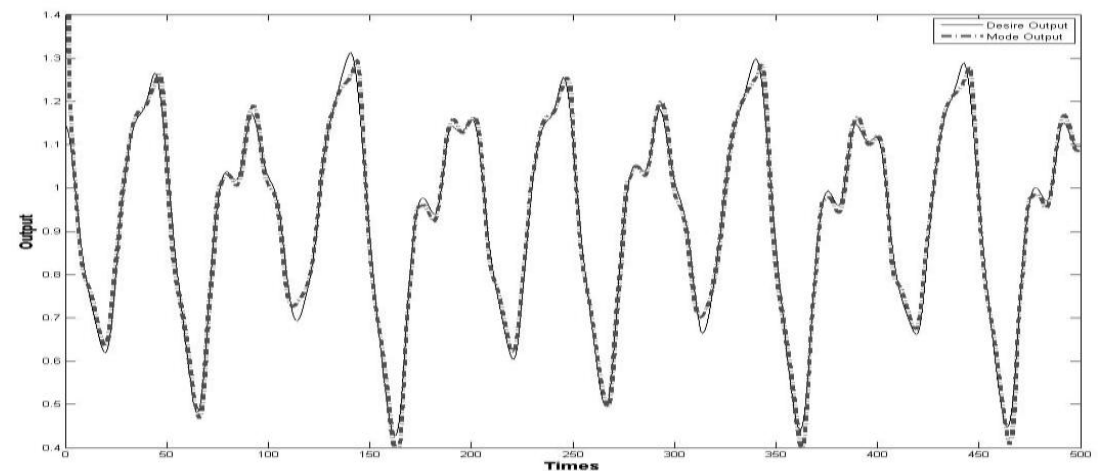

(b)

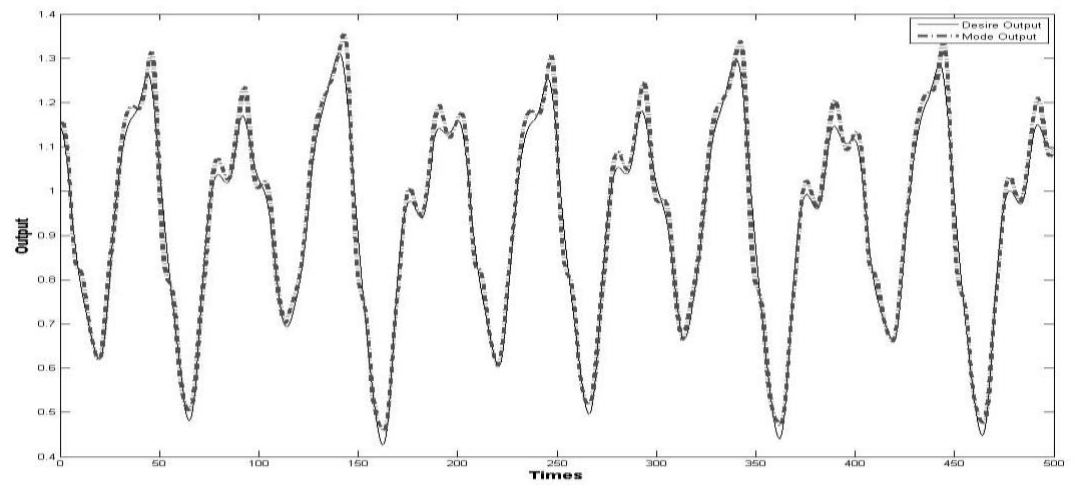

(c)

Fig. 5. Prediction results of (a) the proposed SPSO method; (b) the PSO method; (c) the GA method.

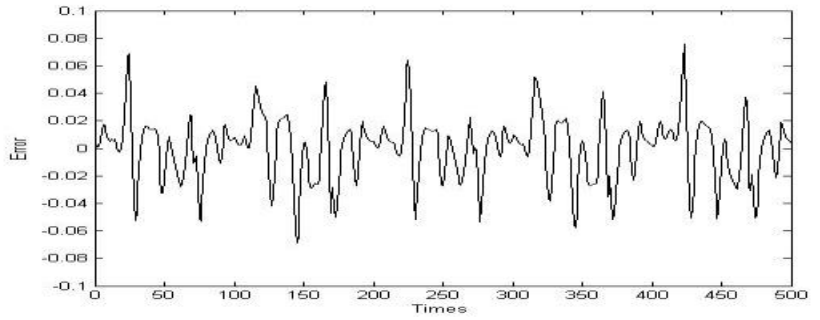

(a)

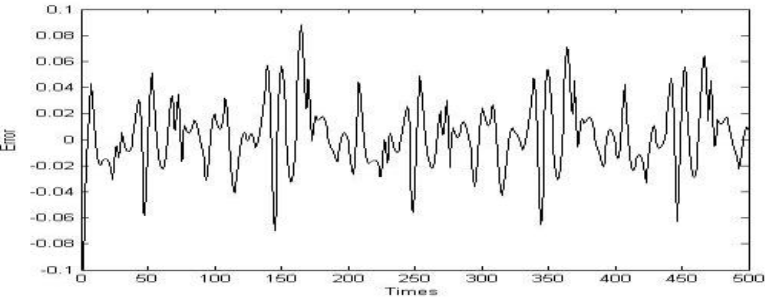

(b) 


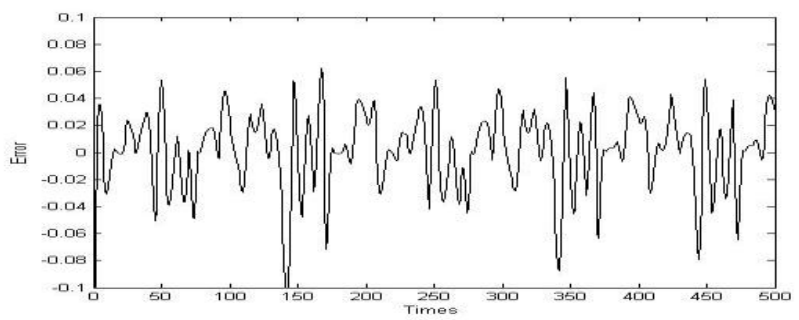

(c)

Fig. 6. Prediction errors of (a) the proposed SPSO method; (b) the PSO method; (c) the GA method.

\section{CONCLUSIONS}

This paper proposed a fuzzy cerebellar model articulation controller (FCMAC) with symbiotic particle swarm optimization (SPSO) for solving prediction problems. An efficient learning algorithm, called symbiotic particle swarm optimization (SPSO), combined the symbiotic evolution and new velocity update function of the modified particle swarm optimization. The proposed learning algorithm can find the global optimal quickly and the good performance more efficiently. Experiment results demonstrated that the converging speed and RMS error of the proposed method is better than other methods.

\section{REFERENCES}

[1] J. S. Albus, "A new approach to manipulator control: The cerebellar model articulation controller," Trans. ASME J. Dyn. Syst., Meas., Contr., pp. 220-227, Sept. 1975.

[2] F. H. Glanz, W. T. Miller, and L. G. Graft, "An overview of the CMAC neural network," in Proc. IEEE Neural Networks Ocean Eng., 1991, pp. 301-308.

[3] E. Mese, "A rotor position estimator for switched reluctance motors using CMAC," Energy Conversion and Management, vol. 44, issue 8 , pp. 1229-1245, May 2003.

[4] D. E. Goldberg, Genetic Algorithms in Search, Optimization and Machine Learning, Addison-Wesley, Massachusetts, 1989.

[5] M. Gen and R. Cheng, Genetic Algorithms and Engineering Design, New York: John Wiley and Sons, 1997.

[6] I. Tazawa, S. Koakutsu, and H. Hirata, "An immunity based genetic algorithm and its application to the VLSI floor plan design problem," in Proc. IEEE International Conference on Evolutionary Computation, Nagoya, 1996, pp. 417-421.

[7] C. J. Lin, H. C. Chuang, and Y. J. Xu, "Face Detection in Color Images Using Efficient Genetic Algorithms," Optical Engineering, vol. 45, no. 4, pp. 047201-1- 047201-12, Apr. 2006.

[8] J. Kennedy and R. Eberhart, "Particle swarm optimization," in Proc. IEEE Int. Conf. on Neural Networks, 1995, pp. 1942-1948.
[9] C. C. Peng and C. J. Lin, "A self-organizing fuzzy CMAC model for classification applications," ICIC Express Letters, vol. 5, no. 7, pp. 2389-2393, July 2011.

[10] D. E. Moriarty and R. Miikkulainen, "Efficient reinforcement learning through symbiotic evolution," Mach. Learn., vol. 22, pp. 11-32, 1996.

[11] C. F. Juang, J. Y. Lin, and C. T. Lin, "Genetic reinforcement learning through symbiotic evolution for fuzzy controller design," IEEE Trans. on Syst., Man, and Cybern. B, vol. 30, no. 2, Apr. 2000.

[12] R. S. Cowder, "Predicting the Mackey-glass time series with cascade-correlation learning," in Proc. Connectionist Models Summer School, 1990, pp. 117-123.

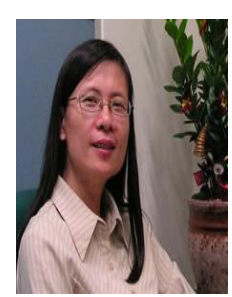

Chin-Ling Lee received the B. A. degree in English from Tamkang University, Taiwan, R. O. C., in 1986, the M. A. degree in English from University of Central Missourri, U. S. A., in 1990, and the Ph. D. degree in industrial education from National Taiwan Normal University, Taiwan, R. O. C. Currently, she is associate professor of International Trade Department, National Taichung University of Science and Technology, Taichung City, Taiwan, R. O. C. Her current research interests are e-learning education, English for specific purposes, and English teaching and learning.

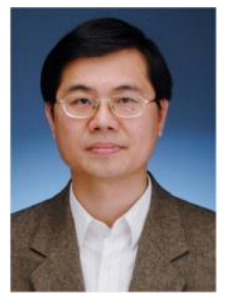

Cheng-Jian Lin received the Ph.D. degrees in electrical and control engineering from the National Chiao-Tung University, Taiwan, R.O.C., in 1996. Currently, he is a Distinguished Professor of Computer Science and Information Engineering Department, National Chin-Yi University of Technology, Taichung County, Taiwan, R.O.C. His current research interests are soft computing, pattern recognition, intelligen control, image processing, bioinformatics, and Android/iPhone program design.

Dr. Lin is an Editorial Board of Applied Computational Intelligence and Soft Computing from 2011, an Editorial Board of Computational Intelligence and Neuroscience from 2011, an Editorial Board of Int'l journal of Control Engineering and Technology from 2011, and an Editorial Board Member of the Open Cybernetics and Systemics Journal from 2010. Dr. Lin is a member of the Phi Tau Phi, the Chinese Fuzzy Systems Association (CFSA), the Chinese Automation Association, the Taiwanese Association for Artificial Intelligence (TAAI), the IEEE Systems, Man, and Cybernetics Society, and the IEEE Computational Intelligence Society. He is an executive committee member of the Taiwanese Association for Artificial Intelligence (TAAI) from 2003 to 2010. He is an executive committee member of the Chinese Fuzzy Systems Association (CFSA) from 2007.

Dr. Lin has received several honors and awards, including the 2006 Outstanding Paper Award of the 11th Conference on Artificial Intelligence and Applications, the 2007 Outstanding Paper Award of the 12th Conference on Artificial Intelligence and Applications, the 2006 Best Paper Award of International Trans. on Computer Science and Engineering (Vol. 32, No. 1), the First Place of the $14^{\text {th }}$ College Contest of IT Services Innovation 2009 , the Silver Prize of International Trade Fair Ideas-Invention-New Productions Nuremberg 2009 (iENA 2009), and the Silver Prize of Seoul International Invention Fair 2010 (SIIF 2010). 